\title{
LA CNAV À L'ÉPREUVE DES RÉFORMES DES RETRAITES
}

entretien avec Pierre Mayeur, réalisé en février 2016 par Samia Benallah, $\underline{\text { Patrick }}$ Aubert

Caisse nationale d'assurance vieillesse | « Retraite et société »

2016/2 N $74 \mid$ pages 187 à 195

ISSN 1167-4687

Article disponible en ligne à l'adresse :

https://www.cairn.info/revue-retraite-et-societe-2016-2-page-187.htm

Distribution électronique Cairn.info pour Caisse nationale d'assurance vieillesse.

(C) Caisse nationale d'assurance vieillesse. Tous droits réservés pour tous pays.

La reproduction ou représentation de cet article, notamment par photocopie, n'est autorisée que dans les limites des conditions générales d'utilisation du site ou, le cas échéant, des conditions générales de la licence souscrite par votre établissement. Toute autre reproduction ou représentation, en tout ou partie, sous quelque forme et de quelque manière que ce soit, est interdite sauf accord préalable et écrit de l'éditeur, en dehors des cas prévus par la législation en vigueur en France. Il est précisé que son stockage dans une base de données est également interdit. 


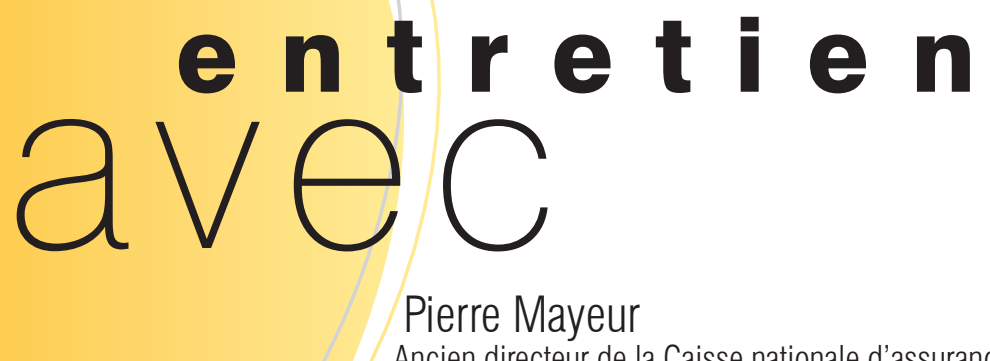

Ancien directeur de la Caisse nationale d'assurance

vieillesse (juillet 2009-mars 2016)

Réalisé en février 2016 par Samia Benallah et Patrick Aubert

\section{La Cnav à l'épreuve des réformes des retraites}

En tant que « concepteur » de réformes, mais également en tant que gestionnaire de caisse de retraite, considérez-vous que les besoins en matière d'évaluation des retraites sont spécifiques par rapport à d'autres politiques sociales, ou plus généralement à d'autres politiques publiques?

Une particularité forte du système des retraites en France, qui est d'ailleurs en train de s'estomper, est qu'il faut partir d'une vision régime par régime. Avant d'engager des réformes des retraites, la puissance publique sollicite en effet la Cnav, afin qu'elle chiffre des mesures pour les salariés du régime général, le service des retraites de l'État pour les fonctionnaires, etc. La Cnav connaît précisément le montant moyen d'une pension versée par le régime général. Si ce montant est de $630 €$, par exemple, cela ne veut pas dire du tout que la personne qui perçoit ce montant vit uniquement avec $630 €$ par mois. En effet, il s'agit seulement de la pension du régime de base.

La question de la retraite est ainsi très fragmentée en France. Il s'agit d'une spécificité du risque vieillesse. En comparaison, lorsqu'on regarde les dépenses de la branche maladie, la Cnamts représente $85 \%$ à $90 \%$ des dépenses. Ce n'est pas le cas de la branche vieillesse, parce que la Cnav n'est que l'un des régimes de base - même si c'est le plus important - et qu'il y a également les régimes complémentaires. Il est donc d'autant plus compliqué de parvenir à des évaluations financières fines, naturellement interrégimes, et d'en comprendre tous les effets économiques, sociologiques, politiques et sociaux. 
La question du « stock » et du « flux » est une autre particularité du domaine des retraites. Une mesure mettra nécessairement des années à monter en charge financièrement, car on ne peut pas remettre en cause le droit qui est servi aux personnes déjà retraitées. Ainsi, lorsqu'on entreprend une réforme, elle ne concernera que les nouvelles générations, c'est-à-dire celles qui sont actuellement actives. Or, celles-ci ont souvent des histoires et un parcours professionnel très différents des générations précédentes. L'erreur serait de les confondre, ce qui est pourtant souvent le cas dans les commentaires politiques en matière de retraite. Par exemple, on insiste régulièrement sur le fait que les jeunes entrent actuellement sur le marché du travail nettement plus tard que les générations partant actuellement à la retraite, ce qui décale mécaniquement l'âge auquel ils peuvent voir le nombre de trimestres requis pour le taux plein. Cette différence est évidente. Cependant, on ne sait absolument pas à quel moment cette génération sortira du marché du travail et si la perte des trimestres constatée en début d'activité sera rattrapée au cours de la carrière ou sur la fin d'activité professionnelle. En somme, il est possible qu'elle compense pour partie cette perte et se retrouve finalement dans une situation similaire à celle de la génération précédente, cette dernière ayant connu des difficultés en fin de parcours professionnel. En bref, en matière de retraite, il est nécessaire d'avoir une vision dynamique.

On constate généralement que les réformes des retraites s'accompagnent d'un besoin de modifications des systèmes d'information vers une unification notamment : est-ce que ces modifications des systèmes d'information sont aussi pensées de manière à permettre de mieux évaluer à terme les effets que vont produire les réformes des retraites?

On a besoin d'avoir une vision agrégée, qui est naturellement interrégimes, et on s'efforce effectivement d'avoir dans notre système d'information de plus en plus cette dimension interrégimes. La Cnav l'a initiée très tôt à travers la base carrière du régime général : le système national de gestion des carrières (SNCC), qui a été l'élément central du droit à l'information et qui est également l'un des éléments de la construction du RGCU (Répertoire général des carrières uniques). Ce nouveau répertoire garantit une vision interrégimes en amont de la liquidation. Le RGCU nous permettra ainsi, à terme, d'avoir une vision globale de l'ensemble du parcours professionnel des assurés. On dispose désormais également de la base EIRR (échanges interrégimes de retraite) : elle est alimentée par tous les régimes, qui la mettent à jour, quasiment en temps réel, pour que l'on puisse avoir une vision exhaustive des avantages de retraite servis à chaque retraité. Grâce à ces échanges, on peut désormais connaître le montant global des pensions que touchent les retraités en France.

C'est une avancée majeure. Par exemple, il était impossible, il y a encore quelques années, de répondre à la question « combien y a-t-il de personnes qui ont moins de $1200 €$ de pension de retraite tous revenus confondus ? », question qui a été posée lors de la mise en place du versement exceptionnel de $40 \operatorname{euros}^{1}$. On pouvait essayer de l'estimer, mais

1. II s'agit du versement d'une prime exceptionnelle de 40 euros, en mars 2015, à tous les retraités relevant d'un régime de retraite obligatoire de base et complémentaire, de droit direct et de droit dérivé, dont le montant total des pensions de retraite n'excédait pas 1200 euros bruts par mois au 30 septembre 2014 (décret $\mathrm{n}^{\circ}$ 2014-1711 du 30 décembre 2014 instituant un versement exceptionnel au bénéfice des titulaires de pensions de retraite inférieures à 1200 euros mensuels). 
ces estimations ne permettaient pas l'ouverture d'un droit individuel. À présent, nous sommes capables de le faire.

\section{Et quand le RGCU verra-t-il le jour?}

Le RGCU sera mis en place progressivement. Une première étape, très importante, est la transformation du SNGC en ce que l'on appelle, le «SNGC + ». Il s'agit, dans le cadre de la liquidation unique entre le régime général, le régime social des indépendants (RSI) et le régime des salariés agricoles (MSA), d'enrichir notre base carrière avec les revenus des artisans et des commerçants. C'est une étape décisive et elle se déroule actuellement.

La seconde étape, très importante pour le RGCU, aura lieu normalement en 2018. Il s'agit de basculer les données sur le RGCU, c'est-à-dire que le module « carrière » de l'Outil retraite ne sera plus utilisé, mais remplacé par un module carrière qui sera directement connecté avec le RGCU. Cela nécessite un calendrier progressif de raccordement des différents régimes sur le RGCU tout au long des années 2018, 2019, 2020 et 2021. Il est aujourd'hui prévu que le dernier régime se connecte sur le RGCU en 2021.

Dans la perspective d'évaluer les effets des réformes des retraites, est-ce que l'accès, pour les chercheurs, à ces données interrégimes, et notamment au RGCU, est envisagé ?

Ces données représenteront en effet une source d'information tout à fait intéressante pour l'évaluation dans le domaine public. Avec le RGCU, on disposera d'une base carrière unique pour les différents régimes de retraite. Les régimes de retraite garderont ainsi leur propre système d'information et de liquidation, mais chaque régime sera « connecté » à une base carrière unique. Il sera ainsi possible de disposer d'une information homogène, diffusée par l'ensemble des régimes de retraites à tous les assurés, quels que soient les régimes dont ils relèvent. C'est d'ailleurs précisément l'un des objectifs de ce projet.

En matière d'évaluation, il s'agit d'une avancée importante : ce que l'on parvient à mesurer aujourd'hui en « bricolant » à partir des différentes sources de données, il sera possible de l'évaluer demain de manière plus fine et rigoureuse grâce à la base carrière unique.

Quant à la diffusion des données aux chercheurs, il n'y a pas de raison d'imaginer que ces données ne soient pas accessibles. Il s'agit toutefois de données brutes et surtout nominatives. Un travail d'anonymisation sera donc préalablement nécessaire.

Vous avez participé à l'élaboration d'une réforme des retraites, celle de 2003. Une des particularités de cette réforme est qu'elle s'appuie très fortement sur les interactions entre les experts et les décideurs, notamment à travers le rôle renforcé du COR dans les décisions concernant l'allongement de la durée d'assurance. Pourquoi ce choix s'est-il imposé en 2003, en particulier?

Je crois pouvoir dire que la réforme de 2003 a été construite sur la base du premier rapport du COR paru en 2001. Quand on le relit, on se rend compte qu'il y a quasiment toute la boîte à outils de la réforme de 2003. L'interaction se fait d'abord dans ce sens. Et la loi de 2003 a conforté l'expertise du COR en lui donnant une consécration législative. 
Par ailleurs, le débat sur les retraites a longtemps souffert de contestations sur l'ampleur des déficits à prévoir. Il y a eu longtemps l'idée qu'en France, il n'y avait pas de problème de financement des retraites mais uniquement un problème de chômage. Un des objectifs, au moment de la création du COR en 2001, était de pouvoir démontrer que, même avec un taux de chômage très favorable (ce qui est par ailleurs évidemment un objectif poursuivi par tous les gouvernements), le problème du financement des retraites restait prégnant. Le premier rapport du COR a pu démontrer que le déséquilibre était avant tout démographique. Le taux de chômage reste bien sûr un paramètre qui peut aggraver les déficits. Il y a d'ailleurs eu des évolutions concernant son chiffrage. Le COR met désormais en place des scénarios avec $7 \%$ de taux de chômage, voire $10 \%$.

La loi de 2003 a ainsi été un prolongement de ce rapport et a renforcé le rôle du COR. L'idée était qu'il devait y avoir une évolution du rapport entre la durée du travail et la durée de retraite et que cette évolution ne devait pas se faire systématiquement de façon défavorable à l'équilibre financier des régimes de retraite. En effet, à durée d'assurance constante, la durée de retraite augmente mécaniquement puisque l'espérance de vie augmente après 60 ans.

Le plan de financement n'a en revanche pas fonctionné : en 2003, on partait du principe que le chômage allait baisser, ce qui aurait permis de basculer des points de cotisations Unedic sur des points retraite, sans augmenter le niveau des prélèvements obligatoires. Malheureusement, cela ne s'est pas vérifié, même si on a pu connaître une période favorable entre 2005 et début 2008, avec un taux de chômage à 7,2 \%.

Pour les réformes suivantes, en 2010 et en 2014, vous étiez Directeur de la Cnav. Celle-ci a été beaucoup sollicitée pour mener des travaux d'évaluation ex ante. Quelles sont vos impressions quant à l'utilisation de toutes ces évaluations?

En période de réformes, il y a en effet un rapport très étroit et plus ou moins direct qui s'établit entre le Cabinet du ou de la ministre et la direction Statistiques, prospective et recherche de la Cnav. C'est un peu la face cachée de la Cnav, en période de réformes. Cette direction fournit des évaluations et fait des scénarios (il y en a eu environ une quarantaine pour la dernière réforme).

Tout cela se fait bien sûr de manière très confidentielle et on n'a jamais eu à regretter ou à noter un moindre défaut de la Cnav en la matière. Dans les phases aiguës de réformes, elle est donc sollicitée et mobilisée. Ce rôle majeur illustre la reconnaissance de l'apport du modèle Prisme ${ }^{2}$ qui permet de simuler les effets d'une augmentation de durée, d'un relèvement de l'âge, etc. C'est intéressant d'avoir vécu ces interactions « de l'autre côté $»$, car on peut se rendre compte que la demande des cabinets n'est pas toujours réaliste. Par exemple, sur la dernière réforme des retraites, l'idée de construire le système sur une combinaison durée/âge, un temps étudiée, a finalement été écartée. Je crois que c'est pour partie grâce à nos analyses qui ont contribué à montrer qu'un tel dispositif - séduisant en apparence - n'était pas en mesure de fonctionner.

2. Pour une présentation détaillée de ce modèle de projection, on peut se référer à l'article de Brossard, Grave et Oliveau dans ce numéro. 
De la même manière, quand on chiffre un relèvement de l'âge ou de la durée d'assurance, il faut également décrire les conséquences que ce décalage peut avoir sur les carrières longues. Ce qui induit certaines interrogations : quelle(s) adaptation(s) pour les carrières longues ? Est-ce qu'on modifie les bornes d'âges ? Est-ce que les carrières longues sont affectées et dans quelle mesure, etc. ? S'installe alors un dialogue entre les services chargés de chiffrer et le décideur politique qui permettra de construire les scénarios. Il y a donc une interaction presque directe qui se crée entre décideurs et experts-évaluateurs.

Bien sûr, il y a des intermédiaires entre le politique (le Cabinet) et les personnes en charge du chiffrage à la Cnav. Il s'agit notamment de la Direction de la sécurité sociale et la Direction de la Cnav. Ces filtres sont très importants pour pouvoir, justement, un peu mieux préciser ce qui est réellement demandé à la Cnav. Mais lorsqu'il y a une accélération du rythme politique, au moment des arbitrages, il faut aller très vite et ces filtres s'effacent alors pour partie. En 2003, nous avons tout de même eu une déconvenue très forte sur les carrières longues parce que le dispositif tel qu'il est apparu le matin du 15 mai 2003, issu d'un accord avec la CFDT, n'avait pas été préalablement chiffré. Personne n'avait imaginé faire commencer les carrières longues à 56 ans ! Dans les projections, nous étions toujours restés sur une ouverture des droits à 58 ou 59 ans pour les gens qui avaient commencé à travailler à 14-15 ans, puis 15-16 ans. Par conséquent, les effets financiers du dispositif carrières longues n'ont pas pu être anticipés correctement : prévoir un dispositif de carrière anticipée à 56 ans n'a évidemment pas le même coût qu'à 58 ou 59 ans, ni le même impact sur le retrait du marché du travail de personnes qui, par ailleurs, étaient justement des seniors actifs. Les effets, diminuer l'âge moyen de liquidation de la retraite au régime général, étaient en outre contradictoires avec l'objectif recherché par la réforme. En bref, même si l'on essaie d'anticiper les effets d'une réforme de manière cohérente, de s'appuyer sur des éléments de chiffrage stabilisés, il arrive un moment où l'essence même des arbitrages politiques ne rend pas la décision toujours très rationnelle.

Le rythme des réformes est de plus en plus soutenu : est-ce que vous avez le sentiment qu'on laisse suffisamment de temps passer entre deux réformes, et surtout que l'on a connaissance de toutes les conséquences des réformes passées?

En termes de prévisions financières, à la Cnav, avec le modèle Prisme, nous avons considérablement progressé et nous sommes de plus en plus précis. Nous avons par exemple évalué parfaitement le dispositif du décret du 2 juillet 2012. Une fois que le dispositif a été définitivement acté, on a su indiquer dès l'automne 2012, donc très rapidement, quel serait le nombre de bénéficiaires en 2013, 2014, etc. Aujourd'hui, sur les dépenses retraites, la précision des prévisions est très fine, avec une marge d'erreur de l'ordre de 50 à 60 millions d'euros, sur une masse de 110 milliards d'euros ${ }^{3}$. Cette précision est remarquable.

Quant aux réformes en elles-mêmes, il y a eu trois grandes lois : 2003, 2010 et 2014. De mon point de vue, on est arrivés au bout de ce genre de dispositifs législatifs. Ils nécessitent la mobilisation des partenaires sociaux et des parlementaires. Ils suscitent de longs débats et des controverses qui se traduisent in fine par des lois avec un trop grand nombre d'articles. Malgré cela, l'insatisfaction domine car toutes les spécificités de la retraite ne

3. Chiffres de 2015. 
peuvent être prises en compte dans un même texte de loi. Le débat sur les retraites est très complexe, et sauf à faire une réforme qui remettrait tout le système à plat - scénario à mon sens illusoire - il est impossible d'envisager une réforme qui réparerait toutes les injustices et prendrait en compte toutes les situations

Je vais prendre deux illustrations qui montrent les inconvénients de cette inflation de réformes. Premièrement, la durée d'assurance requise affichée est très élevée, ce qui peut légitimement faire peur notamment aux jeunes générations qui peuvent considérer que 43 années de cotisation représentent une durée inatteignable. Mais, dans le même temps, on s'ingénie à créer des dispositifs qui permettent de compenser les périodes sans cotisation par des majorations, pour permettre aux assurés de parvenir plus facilement à la durée requise. C'est une des raisons pour lesquelles je pense qu'aujourd'hui, on est arrivés un peu au bout de ce mécanisme de réformes.

Deuxièmement, l'accord sur les retraites complémentaires de 2015 complexifie également grandement le système de retraite. Que l'on soit d'accord ou non avec cet accord, on ne peut qu'admettre qu'il crée une distorsion entre les salariés privés et les assurés relevant d'autres régimes sur le paramètre de l'âge. Et c'est la première fois, à ma connaissance, qu'il y a de manière explicite, un accord des régimes complémentaires créant ce type de distorsion.

Est-ce que les réformes des retraites s'accompagnent nécessairement d'une augmentation de la charge de travail pour les équipes et éventuellement d'une réorganisation?

Les réformes des retraites ont des effets sur le fonctionnement « normal » des caisses de retraite à deux niveaux. Il y a d'abord forcément un besoin d'information qui se crée à chaque réforme des retraites. Cela se traduit par davantage d'assurés qui appellent notre plateforme téléphonique ou regardent notre site internet. Ils demandent un entretien, pas forcément parce qu'ils veulent liquider tout de suite leur retraite, mais ils veulent savoir à peu près dans quelles conditions ils partiront. Ce phénomène augmente la charge de travail, mais répondre à ces interrogations est le cœur de métier des caisses de retraites, il faut donc savoir répondre présent. La difficulté réside plutôt dans la communication autour des dispositifs qui sont en cours d'élaboration. Certaines personnes appellent alors même que le dispositif n'a pas encore été présenté en Conseil des ministres ou adopté au Parlement et qu'on en ignore la forme finale. Ce fut le cas de la réforme de 2010. La Cnav n'était pas historiquement préparée à répondre à ce genre de demandes. Nous avons fait des progrès en la matière et nous essayons à présent $d$ 'informer le plus possible nos propres techniciens, et notamment les personnes qui répondent sur les platesformes téléphoniques, en amont des projets de réforme des retraites.

Le deuxième effet concerne le flux de liquidation des droits à la retraite. Le relèvement de l'âge de départ en retraite à partir de 2011 a bien sûr eu un impact très important sur ces flux. Les flux de liquidations ont toujours été structurellement et naturellement heurtés, mais les périodes étaient toujours les mêmes : un pic important au mois de janvier, puis deux mois de creux et à nouveau un pic important, puis deux mois creux et ainsi de suite. Avec le relèvement progressif de l'âge d'ouverture des droits, on a assisté à une forme de désorganisation de ces flux, qui ont mis en difficulté certaines caisses qui étaient plus fragiles. 
Dans tous les cas, on essaie d'anticiper les difficultés potentielles de mise en œuvre des dispositifs. Notamment, on essaie de se faire entendre, en amont, avant l'adoption des dispositifs afin d'expliquer les difficultés inhérentes à sa mise en place et les conséquences en termes de gestion. Mais les considérations de gestion ne sont pas primordiales au moment de l'élaboration d'une réforme. Je pense qu'au-delà des questions de gestion, j'ai le devoir, en tant que gestionnaire de caisse de retraites, d'attirer l'attention du politique sur la question de l'explication à l'assuré, c'est-à-dire de la lisibilité. C'est peut-être sur ce point qu'il faut mettre l'accent pour influer sur les décisions, en insistant sur le fait qu'un dispositif compliqué pour l'assuré sera également difficile à accepter par ce dernier, et donc à appliquer.

Y a-t-il déjà eu un dispositif issu d'une réforme des retraites qui n'aurait pas, compte tenu des difficultés de sa mise en œuvre, produit les effets escomptés ?

Dans la dernière réforme des retraites, il y a une disposition sur l'affectation des cotisations vieillesse entre deux années civiles ${ }^{4}$ que nous avons jugée particulièrement problématique dès la présentation de l'avant-projet de loi devant le conseil d'administration de la Cnav. Et de fait, la dernière loi de financement de la Sécurité sociale a supprimé ce dispositif5. Par conséquent, nous n'avons pas eu à mettre en application cette mesure, même si elle apparaissait plus équitable pour les assurés aux carrières heurtées. C'est un exemple typique d'un dispositif, certes théoriquement utile, mais impossible à appliquer.

Vous vous apprêtez à quitter la Cnav après sept années à son service. Quelle est votre évaluation personnelle des réformes des retraites qui ont été mises en œuvre? À votre avis, quels sont les éléments d'évaluation les plus lacunaires en matière d'effets des réformes des retraites?

Je ne suis pas certain qu'on ait encore des choses à apprendre dans ce domaine. Je pense plutôt qu'il est désormais temps de communiquer sur les réformes des mises en œuvre et leurs effets. J'ai une vision positive et optimiste car, en matière de retraite, la France a déjà beaucoup réformé. On réforme notre système de retraite depuis 1993, et heureusement cela a eu des conséquences importantes. Il faudra encore certainement des ajustements, mais je ne suis pas du tout persuadé que de nouvelles grandes réformes, prenant la forme d'une loi de l'ampleur de celles de 2003, 2010 ou 2014, soient nécessaires dans l'immédiat.

Ce qui me parait curieux, c'est qu'aucun gouvernement ne communique sur ce bilan positif des réformes, notamment en termes d'économie pour les régimes qui étaient menacés de déficit. Le débat politique reste toujours axé sur la question du régime unique. Je trouve dommage qu'il n'y ait pas cet effort de pédagogie et d'information auprès de la population.

4. II s'agit de l'article 25 alinéa 2 de la loi n 2014-40 du 20 janvier 2014 garantissant l'avenir et la justice du système de retraites qui introduit les modalités d'affectation des cotisations d'assurance vieillesse entre deux années civiles successives pour les assurés qui ne justifient pas, au cours de chacune des années civiles considérées, de quatre trimestres d'assurance vieillesse dans l'ensemble des régimes de retraite légalement obligatoires.

5. Le dispositif a en effet été abrogé par l'article 52 de la loi n 2015-1702 de la loi du 21 décembre 2015. 
Il est vrai que certaines mesures ne sont pas évidentes à assumer, comme le relèvement de l'âge, l'augmentation de la durée d'assurance requise, le fait d'augmenter le nombre d'années de salaire dans le calcul du salaire annuel moyen, l'indexation des pensions, le décalage des dates de revalorisations, etc. Ces mesures ont un impact très important mais pour toutes les réformes des retraites, il y a un point commun rarement mis en valeur : l'injection massive de recettes supplémentaires dans le système.

Je reconnais qu'il n'est pas facile de parvenir à rendre compte clairement des efforts accomplis en matière de retraite. Oui, il est difficile d'être pédagogue lorsqu'il est question d'augmentation de points de cotisations ou de projections de points de PIB. C'est une vraie difficulté.

À l'inverse, il y a un discours de la part des politiques qui me paraît gênant, et un peu facile, s'agissant du manque d'information retraite. Beaucoup d'efforts ont pourtant été déployés depuis 2003 : nous avons créé le droit à l'information en matière de retraite, nous envoyons des relevés individuels de situation (RIS) pour les assurés à partir de 35 ans, des estimations individuelles globales (EIG) à partir de 55 ans. Nous avons créé les entretiens information retraite à partir de 45 ans. Nous avons lancé un projet de portail commun interrégimes $(\mathrm{PCl})$ avec un simulateur, opérationnel à partir de l'automne 2016.

Lorsque j'entends les acteurs politiques expliquer que la situation est catastrophique car personne ne comprend rien à la retraite, j'aurais tendance à nuancer le propos. Bien sûr, notre système est complexe, et l'information est en effet au cœur de nos préoccupations. Mais l'argument consistant à dire que les jeunes d'aujourd'hui ne savent pas ce qu'ils vont percevoir comme retraite est un argument bien faible... parce que cela me paraît plutôt normal ! Comment pourrait-on prévoir la situation de la France dans 40 ans, c'est-à-dire en 2056 ?

J'ai tendance à penser qu'il y a, de ce point de vue, une grande confusion. Certes, l'architecture de notre système est historiquement complexe. Mais il ne faut pas oublier ce qui découle directement de la complexité de la réglementation et de son évolution permanente. Les législateurs en portent forcément la responsabilité. Nous nous efforçons, en tant que régime, de rendre accessibles ces règles et d'informer nos assurés en cas de changement de ces règles. Cette complexité, contrairement à ce qui est affirmé un peu facilement, ne vient pas toujours de la multitude de régimes, mais bien des règles nombreuses et évolutives. En matière de droit à l'information, nous sommes même en avance par rapport à d'autres pays, notamment la Belgique, qui est en train de mettre en place des dispositifs proches de ceux que nous appliquons depuis le milieu des années 2000.

Quel est votre sentiment par rapport aux objectifs d'équité, notamment intragénérationnels ? Pensez-vous que les réformes ont pu, ou vont, aggraver une certaine forme d'iniquité ?

Je crois qu'il y a deux sujets distincts :

- En ce qui concerne l'équité entre régimes, il y a eu des avancées significatives qui ont contribué à homogénéiser les règles des différents régimes de retraite. Plus précisément, on a rendu possible, avec la réforme de 2003 , l'harmonisation de la durée d'assurance entre les régimes, parce que l'on a créé la notion de durée d'assurance tous régimes dans les régimes de la fonction publique, notion n'existait pas auparavant. La montée en charge 
de cette réforme, qui s'est faite sans grandes difficultés et de façon relativement rapide, s'achèvera en 2020. Lorsqu'on regarde les réformes d'harmonisation à l'étranger, et notamment en Italie, on se rend compte que les montées en charge se sont faites de manière très progressive, sur de très longues périodes; cela n'a pas été le cas en France. Le processus d'harmonisation de la durée dans les régimes de la fonction publique, entre 2004 et 2020, est pourtant significatif, avec la mise en place de décotes qui n'existaient pas auparavant. Par ailleurs, l'âge légal a été harmonisé globalement sur les régimes, même s'il reste les catégories actives de la fonction publique. En outre, l'indexation des pensions est également harmonisée entre les régimes depuis 2003. Il reste toutefois la question de la réversion qui est un sujet extrêmement compliqué. Là, une vraie différence subsiste entre les régimes. - En ce qui concerne l'équité intergénérationnelle, j'ai l'impression qu'il n'y a pas de conclusions définitives. Tout dépend des carrières des assurés touchés par les réformes, et notamment des fins de carrière. 